\title{
Embolia Pulmonar em Portugal: Epidemiologia e Mortalidade Intra-Hospitalar
}

\section{Pulmonary Embolism in Portugal: Epidemiology and In-Hospital Mortality}

\author{
Miguel GOUVEIA $\rrbracket^{1}$, Luís PINHEIRO 2,5 , João COSTA $2,3,4$, Margarida BORGES $2,3,6$ \\ Acta Med Port 2016 Jul-Aug;29(7-8):432-440 - http://dx.doi.org/10.20344/amp.6367
}

\section{RESUMO}

Introdução: Em Portugal, a epidemiologia da embolia pulmonar aguda é mal conhecida. Neste estudo, pretendeu-se caracterizar a embolia pulmonar a partir dos dados do internamento hospitalar, assim como avaliar a sua mortalidade intra-hospitalar (definida como mortalidade do internamento hospitalar) e respetivos fatores de prognóstico.

Material e Métodos: Microdados dos Grupos de Diagnóstico Homogéneo dos hospitais do Sistema Nacional de Saúde (2003 a 2013) e dados sobre população do Instituto Nacional de Estatística para estabelecer a evolução dos internamentos, da mortalidade intrahospitalar e das taxas de incidência na população. Os microdados foram estudados numa regressão logit modelizando a mortalidade intra-hospitalar como função de características individuais e de variáveis de contexto.

Resultados: Entre 2003 e 2013 ocorreram 35200 episódios de internamento (doentes $\geq 18$ anos) em que pelo menos um dos diagnósticos foi embolia pulmonar (diagnóstico principal em 67\% dos casos). A taxa de incidência estimada em 2013 foi 35/100 000 habitantes ( $\geq 18$ anos). Entre 2003 e 2013, o número anual de episódios foi aumentando, mas a taxa de mortalidade intra-hospitalar foi diminuindo (de $31,8 \%$ para $17 \%$ em todos os episódios e de $25 \%$ para $11,2 \%$ nos episódio com embolia pulmonar como diagnóstico principal). Entre 2010 e 2013 a probabilidade de morte reduziu-se com a existência de registo de tomografia computorizada, em doentes do género feminino e aumentou com a idade e a presença de comorbilidades.

Discussão: Na última década ocorreu um aumento da incidência de embolia pulmonar provavelmente relacionado com um maior número de pessoas dependentes e acamadas. No entanto, verificou-se uma redução da mortalidade intra-hospitalar de tal dimensão que a própria taxa de mortalidade na população em geral se reduziu. Uma explicação possível é que tenha ocorrido um aumento dos episódios de embolia pulmonar com níveis de gravidade incrementalmente menores, pela maior capacidade de diagnóstico de casos menos graves. Outra explicação possível é uma maior efetividade dos cuidados de saúde hospitalares. De acordo com a análise de regressão logística, as melhorias na efetividade dos cuidados hospitalares nos últimos anos são o principal responsável pela redução da mortalidade.

Conclusão: Cerca de 79\% da redução da mortalidade intra-hospitalar da embolia pulmonar entre 2003 e 2013 pode-se atribuir à maior efetividade dos cuidados de saúde hospitalares e o restante à alteração favorável nas características dos doentes associadas ao risco de morte.

Palavras-chave: Avaliação do Impacto na Saúde; Embolia Pulmonar/epidemiologia; Embolia Pulmonar/mortalidade; Grupos de Diagnósticos Homogéneos; Incidência; Mortalidade Hospitalar; Portugal.

\section{ABSTRACT}

Introduction: In Portugal, the epidemiology of acute pulmonary embolism is poorly understood. In this study, we sought to characterize the pulmonary embolism from the hospital data and evaluate its in-hospital mortality and respective prognostic factors.

Material and Methods: The study used diagnostic related groups data from National Health System hospitals from 2003 to 2013 and National Statistics Institute population data to establish the evolution of admissions with the diagnosis of pulmonary embolism, their inhospital mortality rates and the population incidence rates. Diagnosis-related group microdata were used in a logit regression modeling in-hospital mortality as a function of individual characteristics and context variables.

Results: Between 2003 and 2013 there were 35,200 episodes of hospitalization in patients with 18 or more years in which one of the diagnoses was pulmonary embolism (primary diagnosis in $67 \%$ of cases). The estimated incidence rate in 2013 was $35 / 100,000$ population ( $\geq 18$ years). Between 2003 and 2013, the annual number of episodes kept increasing, but the in-hospital mortality rate decreased (from $31.8 \%$ to $17 \%$ for all cases and from $25 \%$ to $11.2 \%$ when pulmonary embolism was the main diagnosis). The probability of death decreases when there is a computerized tomography scan registry or when patients are females and increases with age and the presence of co-morbidities.

Discussion: In the last decade there was an increased incidence of pulmonary embolism likely related to an increased number of dependents and bedridden. However, there was a in-hospital mortality reduction of such size that the actual mortality in the general population was reduced. One possible explanation is that there has been an increase in episodes of pulmonary embolism with incrementally lower levels of severity, due to the greater capacity of diagnosis of less severe cases. Another possible explanation is greater effectiveness of hospital care. According to the logistic regression analysis, improvements in hospital care effectiveness in recent years are primarily responsible for the mortality reduction.

Conclusion: About $79 \%$ of the reduction of in-hospital mortality of pulmonary embolism between 2003 and 2013 can be attributed to

1. Católica Lisbon School of Business and Economics. Lisboa. Portugal.

2. Centro de Estudos de Medicina Baseada na Evidência. Faculdade de Medicina. Universidade de Lisboa. Lisboa. Portugal.

3. Laboratório de Farmacologia Clínica e Terapêutica. Faculdade de Medicina. Universidade de Lisboa. Lisboa. Portugal.

4. Unidade de Farmacologia Clínica. Instituto de Medicina Molecular. Faculdade de Medicina. Universidade de Lisboa. Lisboa. Portugal.

5. Serviço de Medicina II. Centro Hospitalar Lisboa Norte. Lisboa. Portugal.

6. Unidade de Farmacologia Clínica. Centro Hospitalar Lisboa Central. Lisboa. Portugal.

$\bowtie$ Autor correspondente: Miguel Gouveia. mig@ucp.pt

Recebido: 05 de março de 2015 - Aceite: 07 de março de 2016 | Copyright @ Ordem dos Médicos 2016 
greater effectiveness of hospital care and the rest to the favorable change in patient characteristics associated with risk of death.

Keywords: Diagnosis-Related Groups; Health Impact Assessment; Incidence; Hospital Mortality; Portugal; Pulmonary Embolism/ epidemiology; Pulmonary Embolism/mortality.

\section{INTRODUCÃO}

O tromboembolismo venoso inclui a trombose venosa profunda e a embolia pulmonar (EP) e é a terceira doença cardiovascular mais frequente com uma incidência anual entre 100 a 200 por 100000 habitantes. ${ }^{1}$ A embolia pulmonar pode ser classificada em aguda e crónica. Os doentes com embolia pulmonar aguda desenvolvem sinais e sintomas de obstrução dos vasos pulmonares. ${ }^{2}$ Os doentes com embolia pulmonar crónica desenvolvem um quadro lentamente progressivo de dispneia relacionada com hipertensão pulmonar. ${ }^{3}$

A EP é uma doença comum, frequentemente fatal, ${ }^{4}$ definida como obstrução da artéria pulmonar, ou de um dos seus ramos, por material (trombo, tumor, ar ou gordura) proveniente de um ponto distante do organismo. O risco da EP está aumentado em doentes submetidos a imobilização prolongada, cirurgia nos três meses anteriores, presença de doença maligna, doença cardíaca, doenças auto-imunes e história de tromboembolismo venoso. A EP não tratada está associada a uma taxa de mortalidade de cerca de $15 \%$ aos três meses ${ }^{5}$ e de $25-30 \%$ aos cinco anos, ${ }^{2}$ sendo a EP recorrente uma causa frequente de morte nestes doentes.

Na maioria dos casos, o quadro de apresentação da EP é inespecífico tornando o diagnóstico difícil. ${ }^{6} \mathrm{O}$ diagnóstico rápido e a instituição de terapêutica podem reduzir substancialmente a mortalidade associada. Num estudo realizado nos Estados Unidos da América (EUA), ${ }^{7}$ em que se avaliou mais de 42 milhões de mortes ocorridas ao longo de 20 anos (1979 - 1998), a EP estava presente no certificado de óbito de 600000 doentes (1,5\%), tendo sido a causa primária de morte em 200000 casos. A introdução da tomografia computorizada (TC) torácica na prática clínica $^{3}$ correlaciona-se com uma duplicação da estimativa da incidência da EP (de 62,1 para 112,3 casos por 100000 nos EUA). ${ }^{8}$

Em Portugal, a epidemiologia da EP é mal conhecida. O objetivo primário deste estudo foi caracterizar a EP em internamento hospitalar cobrindo um horizonte de análise de 2003 a 2013. Um segundo objetivo foi avaliar a mortalidade intra-hospitalar da EP, definida como mortalidade do internamento hospitalar, e os seus fatores de prognóstico.

\section{MATERIAL E MÉTODOS}

Para identificar os casos de EP no sistema de saúde português, utilizou-se a base de dados da Administração Central do Sistema de Saúde (ACSS) que é um registo administrativo de episódios hospitalares ocorridos em unidades do Serviço Nacional de Saúde (SNS) em Portugal continental. Não foram contempladas outras hipóteses de EP em internamentos fora do SNS. A informação da ACSS inclui diagnósticos e procedimentos codificados a partir dos processos hospitalares recolhida por médicos codificadores treinados na utilização da Classificação Internacional das Doenças (ICD9-CM).

Foram analisados os episódios de internamento de doentes maiores de 18 anos com alta entre 1 de janeiro de 2003 e 31 de dezembro de 2013, em que a EP, definida pelo código ICD9-CM 415.19 (embolia ou enfarte pulmonar, não classificados noutra parte NCOP), era o diagnóstico principal ou um dos até 19 outros diagnósticos presentes na base de dados. Para calcular a incidência de episódios de internamento com EP, foram obtidas as estimativas da população residente do Instituto Nacional de Estatística, para os anos em estudo.

Para o estudo das comorbilidades da EP utilizou-se o software de classificação clínica (CCS - Clinical Classification Software). Trata-se de uma ferramenta que agrupa um conjunto de diagnósticos e procedimentos codificados pela ICD9-CM num número mais reduzido de categorias com significado clínico. Ou seja, colapsaram-se condições que não tendo exatamente os mesmo diagnósticos têm a mesma natureza e prognósticos semelhantes, permitindo a sua utilização pela análise estatística. Além da caracterização das comorbilidades em geral, considerou-se ser relevante e útil compreender especificamente o papel que doenças crónicas possam ter nestes doentes.

Para identificar e quantificar a presença de doenças crónicas utilizou-se o software Indicador de Condição Crónica ( $\mathrm{CCl}$ - Chronic Condition Indicator). ${ }^{9}$ Esta ferramenta define condição crónica como aquela com duração superior a 12 meses e preenchendo pelo menos um dos seguintes critérios: limitações no autocuidado, na vida independente ou na interação social, necessidade de intervenção médica continuada.

Adicionalmente, utilizou-se o índice de Charlson-Deyo, um índice numérico baseado na presença de um conjunto chave de códigos ICD-9 CM pertencentes a 19 categorias clínicas e que traduz a maior ou menor presença e peso das comorbilidades no caso individual. O índice Charlson-Deyo pode ser utilizado como marcador de complexidade clínica, para além de potencial preditor de mortalidade.

Foi analisada a mortalidade intra-hospitalar nos casos de EP e desenvolvido um modelo explicativo em que as probabilidades de morte foram modelizadas e estimadas a partir de regressões logit. Os resultados destas regressões são normalmente apresentados como odds ratios. Neste trabalho a forma de apresentação dos resultados é diferente, pretendendo-se que a interpretação dos resultados reportados seja semelhante à de coeficientes de modelos de regressão linear. Assim optou-se por um formato exibindo o efeito médio de alterar cada variável, o que permite uma leitura mais intuitiva dos resultados. No caso de variáveis qualitativas o efeito reportado corresponde a passar a variável de 0 para 1 . Para as outras variáveis trata-se da aproximação linear ao modelo, feita na média da amostra. No modelo, a probabilidade de morte em episódio com 
EP é explicada por variáveis individuais e de contexto, que incluíram o índice de comorbilidade de Charlson-Deyo ${ }^{10-12}$ adaptado para utilização recorrendo a dados codificados em ICD9-CM. Os índices de comorbilidade são ferramentas de investigação clínica que efetuam a normalização quantificável de doenças concomitantes e da sua gravidade, permitindo a comparação entre doentes ou grupos de doentes e entre equipas/instituições.

A análise estatística foi efetuada utilizando o package STATA 13 (STATACORP; College Station, TX, USA) e o Microsoft Excel 2010 (Microsoft Corp., Redmond, WA, USA).

\section{RESULTADOS}

\section{A EP em Portugal a partir dos dados do internamento hospitalar}

Entre 1 de janeiro de 2003 e 31 de dezembro de 2013 ocorreram 35200 episódios de internamento nos hospitais do SNS em doentes com 18 ou mais anos em que pelo menos um dos diagnósticos foi o de EP. Destes, 23669 episódios $(67 \%)$ apresentaram a EP como diagnóstico principal. Estes valores correspondem em média a 3200 episódios por ano, nos quais a EP se apresenta como diagnóstico principal em 2152 casos. A demora média dos episódios de EP foi de 12,3 dias (DP = 10,8), tendo diminuído ligeiramente entre 2003 (12,3 dias) e 2013 (11,5 dias). A evolução ao longo do tempo de todos os episódios com EP e dos episódios em que a EP é o diagnóstico principal pode ser vista na Fig. 1. O internamento de doentes com EP tem aumentado ao longo do tempo, quer este esteja codificado como diagnóstico principal, quer como qualquer um dos diagnósticos. A Fig. 1 mostra que entre 2009 e 2010 ocor- reu uma alteração qualitativa nos dados com um aumento pronunciado do número de episódios de EP, ao mesmo tempo que desceu substancialmente o número global de todos os episódios de internamento no SNS.

Para melhor caracterizar os episódios de EP, avaliámos o número de episódios em que a realização de TC torácica (código ICD 9-CM 87.41) tinha sido codificada num dos procedimentos da base de dados (Fig. 2). De salientar, que a codificação da TC torácica não é obrigatória, pelo que poderá ter sido realizada em maior número do que o estimado.

No que diz respeito à distribuição por género, no período em análise, as mulheres representaram $61,2 \%$ dos episódios, sendo que esta proporção se tem mantido praticamente constante ao longo do tempo. Os episódios de EP ocorreram sobretudo em doentes idosos. A média das idades para todos os casos entre 2003 - 2013 é 70 anos (DP = $15,7)$, registando-se uma pequena diferença entre homens (68,9 anos) e mulheres (70,8 anos). Por outro lado, as medianas das idades foram superiores às médias, tendo sido de 74 anos para a população geral (72 anos nos homens e 75 anos nas mulheres). A Fig. 3 mostra a distribuição por idades e género dos doentes internados com um diagnóstico de EP.

As taxas estimadas de episódios de EP por 100000 habitantes revelam um aumento significativo das taxas de incidência da EP ao longo do tempo, quer nos homens, quer nas mulheres, com valores mais elevados no caso das mulheres (Tabela 1). Como seria de esperar, o ressalto no número de casos observado de 2009 para 2010 repercute-se em aumentos substanciais nas taxas de incidência no mesmo período.

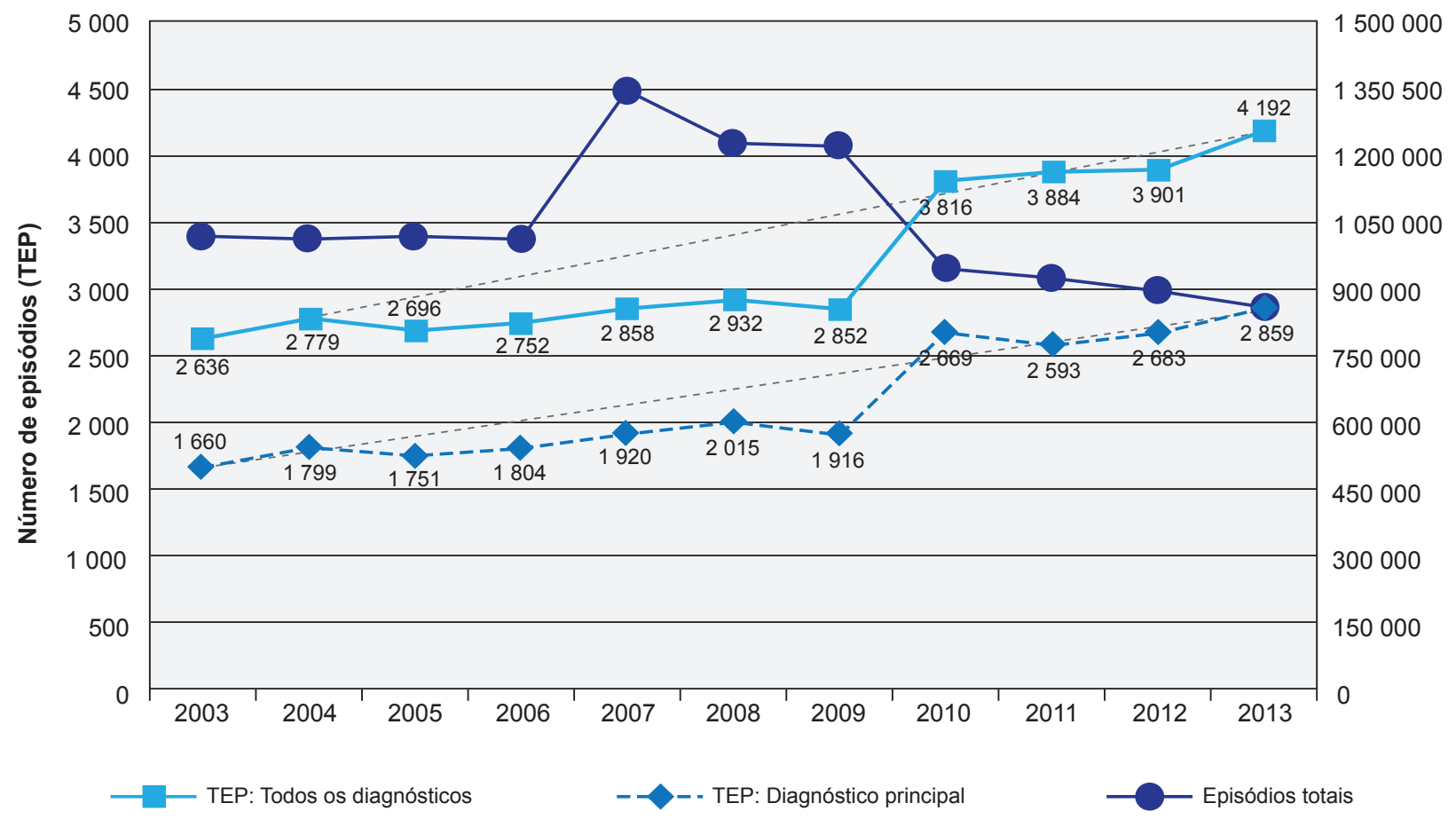

Figura 1 - Episódios de internamento, totais e com o diagnóstico de EP (2003 - 2013) 


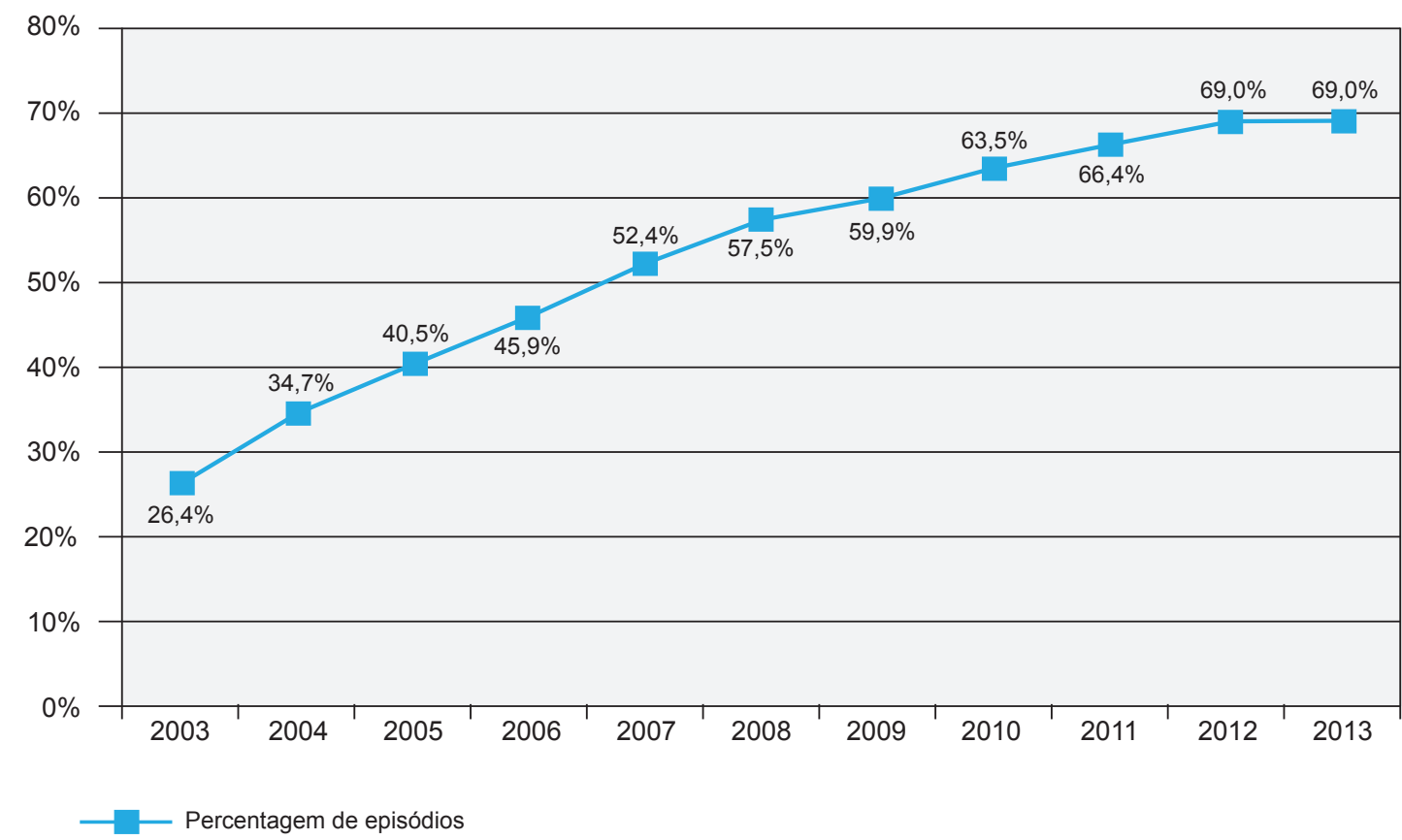

Figura 2 - Proporção episódios de internamento com o diagnóstico de EP e com TC torácica codificada nos procedimentos (2003 - 2013)

O número de comorbilidades, uma variável usualmente associada à gravidade da situação dos doentes, aumentou significativamente (Fig. 4).

Utilizando a classificação CCS Single Level é possível identificar os diagnósticos principais presentes nos doentes com doença cardíaca de origem pulmonar, para além naturalmente da EP. Na Tabela 2 estão patentes os casos dos diagnósticos correspondentes a $1 \%$ ou mais de todos os episódios de internamento. Os diagnósticos principais mais frequentes foram pneumonia, insuficiência cardíaca congestiva, acidente vascular cerebral (AVC), cancro do pulmão, flebite, trombose venosa profunda (TVP) e fratura do colo do fémur. Estas situações ocorreram em 12\% dos casos. Uma comparação entre os anos 2003 a 2009 e os anos 2010 a 2013 mostra que ocorreram alterações na distribuição por CCS Single Level, com aumento do número de diagnósticos de cancro do pulmão de $1,4 \%$ para 1,8\% e uma diminuição do número de diagnósticos de insuficiência cardíaca congestiva de $2,1 \%$ para $1,7 \%$ e da fratura do colo do fémur de $1,2 \%$ para $0,7 \%$.

Os episódios de EP caracterizam-se por uma taxa de mortalidade intra-hospitalar muito alta $(16,8 \%$ no conjunto de todos os anos) com diferenças entre os géneros (18,3\% nos homens e $13,2 \%$ nas mulheres). A taxa de mortalidade intra-hospitalar sofreu uma evolução muito significativa ao longo do tempo decrescendo de $31,8 \%$ em 2003 para $17 \%$ em 2013 em todos os casos com um diagnóstico de EP e de $25 \%$ para $11,2 \%$ nos casos em que EP é o diagnóstico principal (Fig. 5).

Apesar da diminuição da taxa de mortalidade intra-hospitalar, ocorreu um aumento do número de óbitos entre 2009 e 2010 como consequência do aumento do número de episódios. Contudo, entre 2010 e 2013 retomou-se a tendência decrescente. Como seria de prever, a mortalidade intra-hospitalar aumenta com a idade, variando em média entre $7,1 \%$ para o grupo dos 18 aos 29 anos e $32,8 \%$ para o grupo com mais de 79 anos.

\section{A mortalidade intra-hospitalar na EP em Portugal}

Conforme resultados anteriormente apresentados, ocorreu em 2009 um aumento significativo no número de episódios com um diagnóstico de EP. Para diminuir enviesamentos, restringiu-se a análise dos fatores explicativos da mortalidade intra-hospitalar aos anos mais recentes (2010 - 2013) e aos casos em que a EP é o diagnóstico principal. Com estes critérios identificaram-se nos GDH 10 804 episódios de internamentos e 1382 mortes, a que corresponde uma taxa de mortalidade intra-hospitalar média de $12,8 \%$.

Para analisar a mortalidade intra-hospitalar nos episódios de EP utilizou-se um modelo logit onde a probabilidade de morte é explicada por variáveis individuais e de contexto. As variáveis individuais consideradas foram o sexo, idade, idade ao quadrado (ambas as variáveis idade e idade ao quadrado (sobre 10) foram centradas na média para reduzir problemas de multicolinearidade) e o índice de comorbilidade de Charlson-Deyo. Considerámos igualmente a presença das patologias associadas à EP (Tabela 2) como sejam insuficiência cardíaca congestiva, doença cerebrovascular, neoplasias, pneumonia, fratura do colo do fémur. Adicionalmente inclui-se uma variável qualitativa indicando o registo de realização de uma TC torácica. A Tabela 3 mostra os resultados centrais da análise de regressão múltipla logística. 


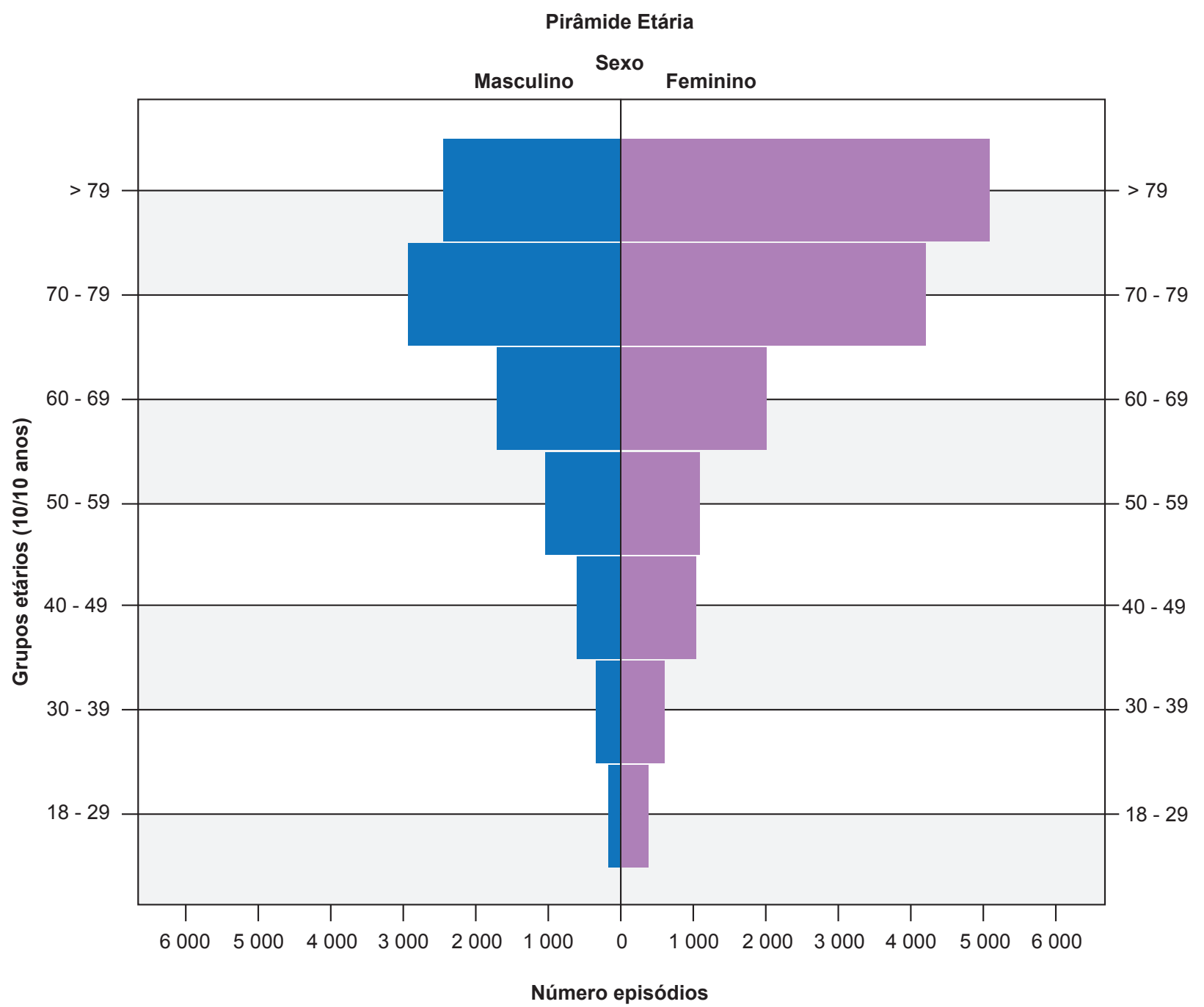

Figura 3 - Distribuição por idades e género dos doentes internados com diagnóstico principal de EP (2003 - 2013)

Tabela 1 - Taxas de incidência de episódios de internamento com um diagnóstico de EP (2003 - 2013)

\begin{tabular}{|c|c|c|c|}
\hline \multirow[b]{2}{*}{ Ano } & \multicolumn{3}{|c|}{ Taxas de incidência por 100000 habitantes } \\
\hline & Homens & Mulheres & Global \\
\hline 2003 & 16,7 & 24,1 & 20,6 \\
\hline 2004 & 19,1 & 25,1 & 22,2 \\
\hline 2005 & 18,9 & 24,0 & 21,6 \\
\hline 2006 & 19,0 & 25,0 & 22,2 \\
\hline 2007 & 18,0 & 28,4 & 23,5 \\
\hline 2008 & 20,0 & 28,7 & 24,6 \\
\hline 2009 & 19,4 & 26,8 & 23,3 \\
\hline 2010 & 26,0 & 38,1 & 32,4 \\
\hline 2011 & 25,8 & 36,5 & 31,5 \\
\hline 2012 & 26,1 & 38,5 & 32,7 \\
\hline 2013 & 29,3 & 40,0 & 35,0 \\
\hline
\end{tabular}

Fonte: Cálculos dos autores com base nos GDH e INE
Ser mulher tem um efeito protetor: em igualdade de circunstâncias relativamente às outras variáveis, uma mulher tem em média uma probabilidade de morte inferior à dos homens em $1,9 \%$. O efeito da idade na probabilidade de morte é também relevante, mas não linear, já que o termo quadrático também é estatisticamente significativo. Definindo a idade média na amostra, 70 anos, como ponto de referência, e mantendo as outras variáveis fixas, uma idade de 55 implica uma redução da probabilidade de morte de $3,5 \%$, ao passo que uma idade de 85 implica um aumento dessa probabilidade de $5,9 \%$.

Um segundo conjunto de coeficientes mede os efeitos das variáveis relativas ao estado de saúde de cada doente. O score de comorbilidade de Charlson-Deyo tem um coeficiente positivo e estatisticamente significativo. Um aumento deste score de um desvio padrão leva a um aumento da probabilidade de morte de $3 \%$. Para o conjunto de patologias estudadas, os coeficientes são significativamente positivos, sendo que cada coeficiente apresentado é já uma medida aproximada do efeito na taxa de mortalidade intra-hospitalar. Os efeitos com maior dimensão são os decorrentes da presença de fratura do colo do fémur e de 


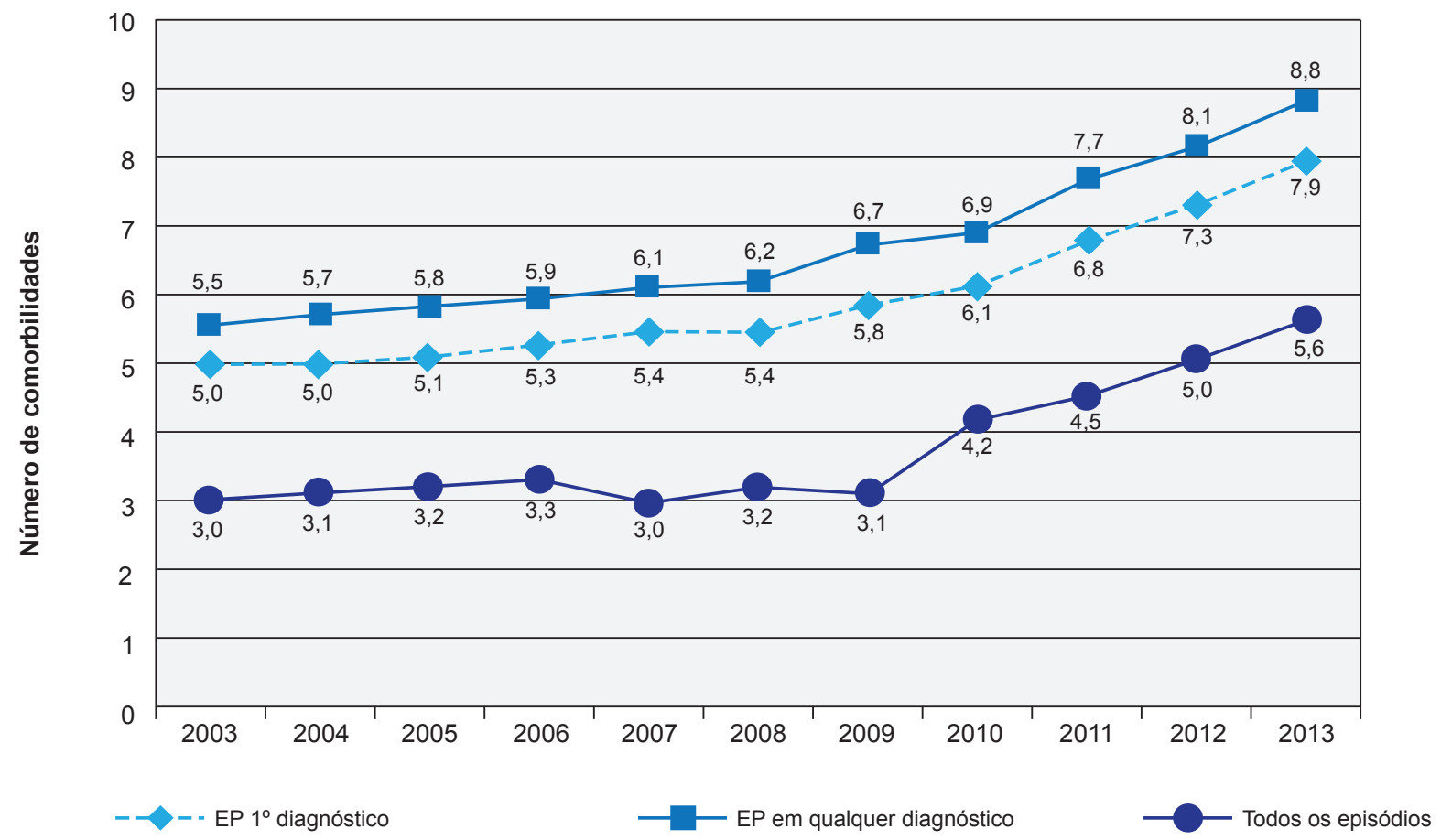

Figura 4 - Número médio de comorbilidades nos episódios de internamento com um diagnóstico de EP e comparação com comorbilidades na totalidade dos internamentos nacionais $(2003$ - 2013)

Tabela 2 - Frequências das principais CSS Single Level como diagnóstico principal em episódios de internamento com um diagnóstico de EP

\begin{tabular}{lcccc} 
& \multicolumn{2}{c}{2003 a 2009} & \multicolumn{2}{c}{2010 a 2013} \\
& Número de & $\begin{array}{c}\text { Episódios } \\
\text { dos Episódios } \\
\text { Totais }\end{array}$ & $\begin{array}{c}\text { Número de } \\
\text { Episódios }\end{array}$ & $\begin{array}{c}\text { dos Episódios } \\
\text { Totais }\end{array}$ \\
\hline Pneumonia & 858 & $4,4 \%$ & 712 & $4,5 \%$ \\
Insuficiência cardíaca congestiva (não hipertensiva) & 411 & $2,1 \%$ & 264 & $1,7 \%$ \\
Doença cerebrovascular aguda & 371 & $1,9 \%$ & 265 & $1,7 \%$ \\
Cancro do pulmão & 267 & $1,4 \%$ & $1,8 \%$ \\
Flebite; tromboflebite e tromboembolismo & 199 & $1,0 \%$ & 172 & $1,1 \%$ \\
Fratura do colo do fémur & 229 & $1,2 \%$ & 117 & $0,7 \%$ \\
\hline
\end{tabular}

pneumonia, que aumentam a probabilidade de morte em $11,5 \%$ e $7,8 \%$ respetivamente.

Por último, uma variável com poder explicativo é o indicador de registo de realização de TC torácica. Em média o registo de TC está associado a um decréscimo na probabilidade de morte de $12 \%$.

\section{DISCUSSÃO}

A incidência de episódios de EP tem vindo a aumentar em Portugal nos últimos dez anos, sendo atualmente estimada em 35 por 100000 habitantes e maior nas mulheres do que nos homens. Este aumento tem sido progressivo, pese embora tenhamos identificado um aumento mais abrupto do número de casos observados de 2009 para 2010. Ou seja, os dados indicam a existência de duas fases distintas na evolução da incidência, a primeira até 2009 e a segunda entre 2010 e 2013.

Para diminuir o risco de enviesamento, a análise dos fatores explicativos da mortalidade intra-hospitalar foi restrita aos anos mais recentes, até porque os autores não identificaram nenhuma razão óbvia para este aumento, nomeadamente alteração das regras de codificação ou um aumento proporcional no registo do número de TC torácicas com consequente aumento do número de casos diagnosticados.

Em muitos episódios de EP estavam presentes comorbilidades como pneumonia, insuficiência cardíaca congestiva, AVC, neoplasias e fratura do colo do fémur. A taxa de mortalidade intra-hospitalar dos episódios de EP é muito elevada e maior nos homens, tendo-se, no entanto, verificado uma evolução muito positiva ao longo do tempo decrescendo de 31,8\% em 2003 para 17\% em 2013 se 


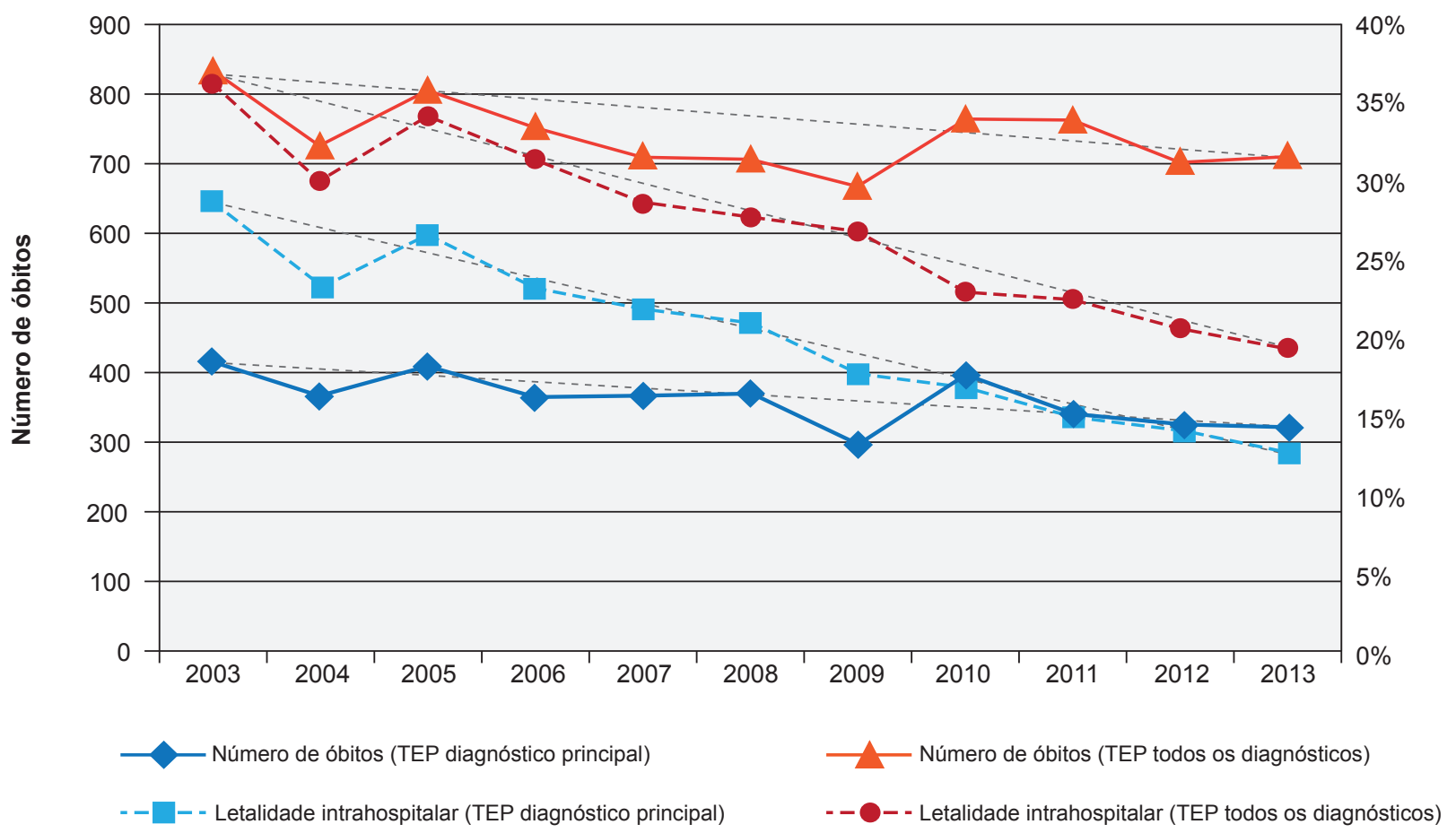

Figura 5 - Óbitos e mortalidade intra-hospitalar dos episódios de internamento com um diagnóstico de EP (2003 - 2013)

considerarmos todos os episódios com um diagnóstico de EP. Para efeitos de referência, podemos adicionar informação sobre as taxas de mortalidade por EP na população em geral de 18 e mais anos. A taxa de mortalidade desceu de 10,4 por 100000 habitantes em 2003 para 8,7 em 2013. Ou seja, apesar do crescimento da incidência, a redução da mortalidade intra-hospitalar foi de tal dimensão que a própria taxa de mortalidade na população em geral se reduziu. Se nos restringirmos aos casos em que a EP é o diagnóstico principal a redução da mortalidade por 100000 habitantes foi ainda mais pronunciada, de 8,2 para 5,7 no mesmo intervalo.

Com referência à análise da mortalidade entre $2010 \mathrm{e}$ 2013, uma explicação possível da redução da mortalidade intra-hospitalar nos episódios com diagnóstico de EP é que tenha ocorrido um aumento dos episódios de EP com níveis de gravidade incrementalmente menores, nomeadamente pela capacidade de diagnosticar casos menos graves com recurso à TC torácica. De facto, na análise por regressão logística, a TC torácica está associada de forma independente a uma redução significativa do risco de morte.

Este resultado pode ser interpretado de várias formas. Uma possibilidade é que a utilização da TC tenha permitido o diagnóstico de EP em casos menos graves e daí a redução da probabilidade de morte. Outra possibilidade é que a TC tenha permitido diagnósticos mais precisos e atempados levando a melhoramentos no tratamento dos doentes com EP.

A estimativa da regressão logística permite compreender melhor as razões da redução da mortalidade hospitalar ao longo do tempo. Esta redução poderia ocorrer devido à redução do nível de gravidade médio dos episódios hospitalares de EP, ou seja devido a alterações favoráveis nas características dos doentes ao longo do tempo. Alternativamente, a redução da mortalidade intra-hospitalar poderia

Tabela 3 - Regressões da mortalidade: efeitos no período 2010 2013

\begin{tabular}{|c|c|c|}
\hline \multirow[t]{2}{*}{ Variável } & \multicolumn{2}{|c|}{ Estimativas } \\
\hline & Efeito Linearizado & Erro Padrão \\
\hline Mulher & $-0,0188^{\star *}$ & 0,0063 \\
\hline Idade & $0,0032^{* *}$ & 0,0002 \\
\hline Idade $^{2}$ & $0,0005^{\star *}$ & 0,0001 \\
\hline Score Charlson-Deyo & $0,0154^{* *}$ & 0,0016 \\
\hline Neoplasia & $0,0187^{*}$ & 0,0085 \\
\hline $\begin{array}{l}\text { Insuficiência cardíaca } \\
\text { congestiva }\end{array}$ & $0,0163^{*}$ & 0,0075 \\
\hline $\begin{array}{l}\text { Doença } \\
\text { cerebrovascular }\end{array}$ & $0,0319^{* *}$ & 0,0097 \\
\hline Pneumonia & $0,0781^{* *}$ & 0,0081 \\
\hline Fratura colo fémur & $0,1158^{*}$ & 0,0554 \\
\hline Registo TC torácica & $-0,1205^{\star *}$ & 0,0060 \\
\hline Observações & 10804 & \\
\hline Pseudo $\mathrm{R}^{2}$ & 0,1315 & \\
\hline Log Verosimilhança & $-3588,07$ & \\
\hline
\end{tabular}

TC: Tomografia computorizada.

Coeficientes podem ser interpretados como numa regressão linear. Análise inclui controlos por ano e região de saúde; ${ }^{*} p<0,05 ;{ }^{* *} p<0,001$. 
dever-se a melhorias na eficácia dos cuidados hospitalares no tratamento da EP. O modelo permite identificar separadamente estes dois efeitos.

Para compreendermos melhor a primeira explicação vale a pena ver exemplos de comorbilidades específicas. $\mathrm{Na}$ amostra em análise, a proporção de episódios com pneumonia diminuiu de $12,1 \%$ em 2010 para $10,1 \%$ em 2013. Usando o modelo, essa redução teve um efeito estimado de reduzir a taxa de mortalidade intra-hospitalar em cerca de $0,15 \%$, aproximadamente $4 \%$ da redução de $3,7 \%$ de facto ocorrida. No entanto, ocorreram alterações noutras variáveis que conduziram a aumentos de gravidade dos episódios. Tal é o caso do índice de comorbilidades de Charlson-Deyo, que cresce de 1,28 para 1,46. Se nada mais se tivesse alterado, este aumento do Charlson-Deyo levaria a um aumento da mortalidade intra-hospitalar ligeiramente inferior a 0,3\%. Estes dois exemplos mostram a necessidade de se fazer uma análise global.

Utilizando o modelo pode estimar-se qual o efeito que se deve à alteração do conjunto das características dos doentes e qual o efeito que se obtém por melhorias de efetividade dos cuidados, assumindo que as características dos doentes não se teriam alterado. O resultado desta decomposição é que a redução de $3,7 \%$ da taxa de mortalidade intra-hospitalar entre 2010 e 2013 se deve numa parcela de $0,8 \%$ a alterações globalmente favoráveis nas características dos doentes e numa parcela de 2,9\% a melhorias no tratamento. Em suma, $21 \%$ da redução da mortalidade deve-se à menor gravidade dos episódios em média e $79 \%$ deve-se a melhorias nos cuidados hospitalares da EP.

\section{CONCLUSÃO}

Os resultados no presente estudo indicam que ocorreu um crescimento da incidência da embolia pulmonar aguda ao longo dos últimos dez anos. O aumento da longevidade, bem como um maior número de pessoas dependentes e acamadas, são fatores a ter em conta para explicar este aumento.

Entre 2009 e 2010 há um acréscimo de casos muito acima da tendência para o qual não se encontra uma explicação evidente. A taxa de incidência estimada para Portugal em 2013, baseada nos dados hospitalares, é de 35 por 100 000 habitantes com 18 ou mais anos de idade. Este valor é inferior ao de outros países, por exemplo à taxa estimada para os EUA, de 112 por 100000 habitantes. Esta diferença poderá ser explicada por eventuais diferenças na incidência real da embolia pulmonar aguda mas não se deverá pôr de parte a possibilidade de esta diferença se dever a uma maior taxa de subdiagnóstico em Portugal por comparação com outros países.

Outra característica marcante da evolução da embolia pulmonar é a mortalidade intra-hospitalar associada que tem vindo a diminuir de forma assinalável, passando de 25\% em 2003 para 11,2\% em 2013 considerando os episódios com um diagnóstico principal de embolia pulmonar. Uma análise baseada num modelo de regressão logística para a mortalidade intra-hospitalar entre 2010 e 2013, em casos com embolia pulmonar como diagnóstico principal, permitiu decompor a evolução da mortalidade intra-hospitalar em dois fatores. O primeiro, as características dos doentes, sumariando a gravidade dos casos. O segundo fator representa a efetividade dos cuidados de saúde hospitalares no diagnóstico e tratamento da embolia pulmonar. A análise revelou que $79 \%$ da redução da mortalidade intra-hospitalar se deve à maior efetividade dos cuidados de saúde hospitalares.

\section{PROTECÇÃO DE PESSOAS E ANIMAIS}

Os autores declaram que os procedimentos seguidos estavam de acordo com os regulamentos estabelecidos pelos responsáveis da Comissão de Investigação Clínica e Ética e de acordo com a Declaração de Helsínquia da Associação Médica Mundial.

\section{CONFIDENCIALIDADE DOS DADOS}

Os autores declaram ter seguido os protocolos do seu centro de trabalho acerca da publicação dos dados de doentes.

\section{CONFLITO DE INTERESSES}

Os autores declaram não ter nenhum conflito de interesses relativamente ao presente artigo.

\section{FONTES DE FINANCIAMENTO}

Este trabalho recebeu apoio da Bayer Portugal através de um Unrestricted Grant à Associação para a Investigação e Desenvolvimento da Faculdade de Medicina. A Bayer Portugal não participou em nenhuma das etapas do projeto e o financiamento não foi condicionado à obtenção de nenhum tipo específico de resultado.

\section{REFERÊNCIAS}

1. Konstantinides SV, Torbicki A, Agnelli G, Danchin N, Fitzmaurice D, Galiè N, et al. 2014 ESC guidelines on the diagnosis and management of acute pulmonary embolism. Eur Heart J. 2014;35:3033-69.

2. Morris TA. Why acute pulmonary embolism becomes chronic thromboembolic pulmonary hypertension: clinical and genetic insights. Curr Opin Pulm Med. 2013;19:422-9.

3. Piazza G, Goldhaber SZ. Chronic thromboembolic pulmonary hypertension. N Engl J Med. 2011;364:351-60.

4. den Exter PL, van der Hulle T, Lankeit M, Huisman MV, Klok FA. Long-term clinical course of acute pulmonary embolism. Blood Rev. 2013;27:185-92.

5. Goldhaber SZ, Visani L, De Rosa M. Acute pulmonary embolism: clinical outcomes in the International Cooperative Pulmonary Embolism Registry (ICOPER). Lancet. 1999;353:1386-9.

6. Huisman MV, Klok FA. How I diagnose acute pulmonary embolism. Blood. 2013;121:4443-8

7. Horlander KT, Mannino DM, Leeper KV. Pulmonary embolism mortality in the United States, 1979-1998: an analysis using multiple-cause mortality data. Arch Intern Med. 2003;163:1711-7.

8. Wiener RS, Schwartz LM, Woloshin S. Time trends in pulmonary embolism in the United States: evidence of overdiagnosis. Arch Intern Med. 2011;171:831-7.

9. HCUP Chronic Condition Indicator. Healthcare Cost and Utilization Project (HCUP). November 2011. Agency for Healthcare Research and 
Quality, Rockville, MD. [consultado 2015 fev 27]. Disponível em: www. hcup-us.ahrq.gov/toolssoftware/chronic/chronic.jsp.

10. Charlson ME, Pompei P, Ales KL, MacKenzie CR. A new method of classifying prognostic comorbidity in longitudinal studies: development and validation. J Chronic Dis. 1987;40:373-83.

11. Deyo RA, Cherkin DC, Ciol MA. Adapting a clinical comorbidity index for use with ICD-9-CM administrative databases. J Clin Epidemiol. 1992;45:613-9.

12. Quan H, Li B, Couris CM, Fushimi K, Graham P, Hider P, et al. Updating and validating the Charlson comorbidity index and score for risk adjustment in hospital discharge abstracts using data from 6 countries. Am J Epidemiol. 2011;173:676-82. 